\title{
Theoretical Calculation of the Circular Dichroism of Unordered Polypeptide Chains
}

\author{
E. W. RONISH and S. KRIMM, Harrison M. Randall Laboratory of \\ Physics and Macromolecular Research Center, University of \\ Michigan, Ann Arbor, Michigan 48104
}

\begin{abstract}
Synopsis
A calculation has been done of the circular dichroism (CD) spectrum of an unordered polypeptide chain. This has been based on a Boltzmann averaging over a dipeptide conformational CD map. This is shown to be valid by comparing the CD spectra of 28-mer oligopeptides with those generated by summing dipeptide CD spectra. The calculated CD spectrum of an unordered polypeptide chain is found to agree with the assignment proposed by Tiff any and Krimm from experimental studies.
\end{abstract}

\section{INTRODUCTION}

A true understanding of the circular dichroism (CD) spectra of polypeptides and proteins must rest ultimately on the ability to correlate the observed spectrum with that calculated for a relevant structural model from a valid theory. A satisfactory start in this direction has been made in recent years for regular structures such as the $\alpha$-helix ${ }^{1,2}$ and the $\beta$ conformation, ${ }^{2-5}$ as well as for certain dipeptides. ${ }^{6,7}$ The situation is less clear for the case of unordered polypeptide chain structures.

The situation with respect to unordered chains is complicated for two reasons. First, the validity of calculations for determining the CD of unordered polypeptide chains ${ }^{8-10}$ may still be questionable. Second, and probably more important, there continues to be disagreement over the experimental CD spectrum which is to be taken as representative of an unordered chain. Although the spectra of charged poly-L-glutamic acid or charged poly-L-lysine have been assumed" to be that of a "random coil," it has been pointed out ${ }^{12}$ that such structures are not valid models because of the likelihood that the side chain charges stabilize a preferred extended helical conformation of the backbone. The geometry of this structure was determined by conformational energy calculations ${ }^{\mathbf{1 3}}$ to be close to a threefold lefthanded helix. This structural assignment of the observed CD spectrum of charged chains has since been supported by spectral studies of uncharged chains known to have an analogous conformation. ${ }^{14}$ The CD spectrum of an unordered polypeptide chain was, partly on this basis, associated with that of poly-L-proline in concentrated aqueous $\mathrm{CaCl}_{2}$ solu- 
tion, ${ }^{15}$ an assignment which was strongly supported by an extended study of a variety of synthetic polypeptides, fibrous proteins, and globular proteins. ${ }^{16}$ The latter $\mathrm{CD}$ spectrum, in contrast to that of the extended helix, has no positive band near $220 \mathrm{~nm}$ but decreases monotonically from a value of $\Delta \epsilon=0$ near $240 \mathrm{~nm}$ to a negative band centered near $200 \mathrm{~nm}$ (depending on the svstem) and of variable intensity ( $\Delta \epsilon \cong-1$ to -4$)$. Subsequent detailed experimental investigations ${ }^{17}$ tend to confirm this assignment.

In order to shed further light on this problem we have done a theoretical calculation of the $\mathrm{CD}$ spectrum of an unordered polypeptide chain. This calculation differs in several details from those previously done, ${ }^{8-10}$ and we believe represents an improvement over them.

\section{METHOD OF CALCULATION}

Our calculation is based on the monopole approximation to the far ultraviolet transitions of the peptide group, a method which seems to have at least a qualitative reliability. ${ }^{1,6,18,21}$ This calculation uses an SCF wave function for the peptide monomer ground state and the $n \pi^{*}$ and $\pi \pi^{*}$ transitions. The polypeptide wave function is constructed from this monomer wave function by configuration interaction.

The $n \pi^{*}$ and $\pi \pi^{*}$ transitions are both included in the secular determinant rather than treating the $\pi \pi^{*}$ by an exciton calculation and then adding in the $n \pi^{*}$ as a perturbation. By including both transitions the calculation becomes more difficult numerically, but it is conceptually simpler and seems to lead to more reliable results. ${ }^{1}$ The $n \sigma^{*}, n^{\prime} \pi^{*}$, and $N V_{2}$ transitions are very poorly understood, and they have not been included. Of course, if the $n \sigma^{*}$ transition lies close to the $\pi \pi^{*}$ transition, for which there may be some evidence, ${ }^{22}$ then it should be taken into account. Since the assignment of the $n \sigma^{*}$ transition is so uncertain, however, we feel that its inclusion would be essentially arbitrary at this time.

The secular determinant of the polypeptide wave function is estimated by a monopole approximation where point charges are placed on or near atoms of the chain. The magnitudes and positions of the monopoles are estimated from the SCF wave function for the monomer. We have selected monopoles which optimize the agreement between observed and calculated CD spectra of a known polypeptide chain structure, viz., the righthanded $\alpha$-helix. These monopoles combine features of those due to Woody ${ }^{1}$ and those due to Bayley et al. ${ }^{6}$ Our monopoles differ from Woody's by having $n \pi^{*}$ charges on the carbon as well as on the oxygen of the carbonyl group, and by not splitting the amide ground state monopoles in two. The first difference seems justified from the results of recent SCF calculations, ${ }^{20}$ the second was necessary in order to prevent unusual proximity of monopoles in the $\alpha$-helix. We have, however, assumed the $\pi \pi^{*}$ monopoles to be split, there being theoretical justification for this since they describe a transition localized in the pi electrons only. The monopoles which we used (for a poly-L-alanine chain) are given in Table I. They are the same as 
TABLE I

Monopoles and Parameters Used in the Calculation

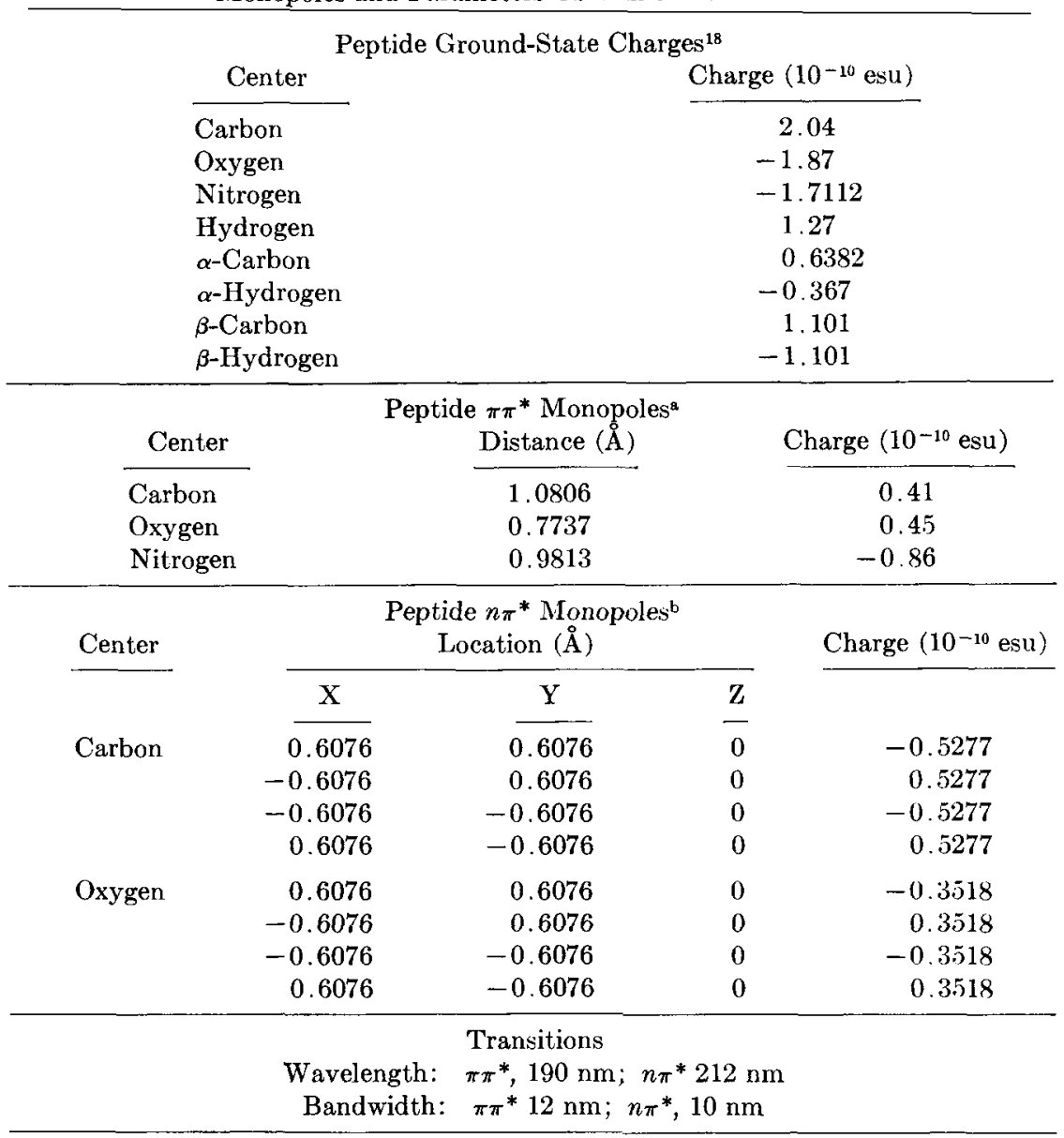

a Equal charges are placed above and below the plane of the peptide group, centered over the $\mathrm{C}, \mathrm{O}$, and $\mathrm{N}$ atoms. The $\pi \pi^{*}$ electric transition dipole moment is $3.05 \mathrm{D},{ }^{20}$ forming an angle of $9^{\circ}$ toward the $\mathrm{C}$ atom from the $\mathrm{N}-\mathrm{O}$ line. ${ }^{18}$

b Quadrupole symmetry is taken around the $\mathrm{C}$ and $\mathrm{O}$ atoms. Coordinates are in the standard coordinate system, with $\mathrm{Z}$ along the $\mathrm{C}$ to $\mathrm{O}$ line and $\mathrm{X}$ in the plane of the peptide group. ${ }^{18}$ The $n \pi^{*}$ magnetic transition dipole mement is $1.1506 \mathrm{BM},{ }^{20}$ pointing from O to C.

those of Bayley et al., except that we have added peptide ground-state monopoles ${ }^{18}$ and we have used semiempirical screening coefficients. ${ }^{1}$

The CD spectrum of a 20 -monomer righthanded $\alpha$-helix of poly-Lalanine was calculated using the monopoles and parameters given in Table I. This spectrum is compared with the experimental spectrum ${ }^{23}$ in Figure 1. The agreement is seen to be satisfactory. It should be noted that in this calculation the $n \pi^{*}$ transition, which on the basis of a study of simple amides in solution ${ }^{19}$ would be at $212 \mathrm{~nm}$, has been taken to be at $220 \mathrm{~nm}$. 


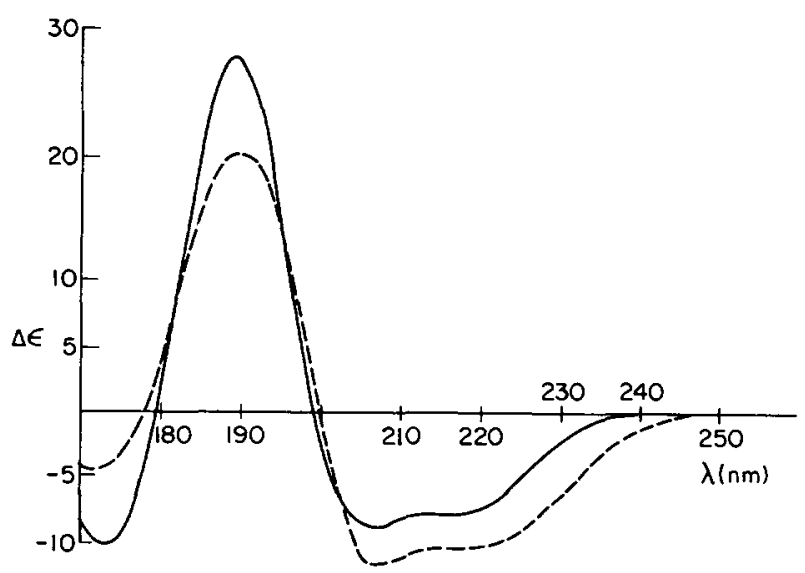

Fig. 1. CD spectrum of the righthanded poly-L-alanine $\alpha$-helix: - , calculated for $\varphi=-47^{\circ} \psi=-57^{\circ}$, using monopoles and parameters of Table I; - - - experimentally observed. ${ }^{22}$

This is thought to be a consequence of the shielding of the carbonyl group in the $\alpha$-helix from the water solvent. ${ }^{19}$ Since no such shielding is expected for the case of the unordered chain, we have placed the $n \pi^{*}$ transition at $212 \mathrm{~nm}$ in these calculations.

\section{APPLICATION TO UNORDERED POLYPEPTIDE CHAIN}

Since the monopoles and parameters of Table I account very well for the CD spectrum of the $\alpha$-helix, we have adopted them for the calculation of the unordered chain. The CD of an unordered chain was taken to be a Boltzmann distribution over the conformational energy map of a dipeptide, the CD of the dipeptides as a function of $\varphi$ and $\psi$ being calculated according to the methods of the previous section. In order to establish the validity of this approach, the CD spectra of several arbitrary oligopeptide structures were calculated and compared with spectra computed as a sum of dipeptide contributions. The results of these preliminary calculations will now be discussed.

The calculations of the dipeptide CD spectra were done at $10^{\circ}$ intervals in $\varphi$ and $\psi$ for the entire range of $\varphi, \psi$ angles. It is of interest to note that the resulting spectra fall into a relatively small number of types, thus permitting a plot of a dipeptide conformational CD map. We have classified these spectra according to the following scheme, which is illustrated by specific examples in Figure 2.

Type 1: A net negative CD at wavelengths greater than $215 \mathrm{~nm}$ followed by a positive $\mathrm{CD}$ with a maximum at a wavelength greater than $187 \mathrm{~nm}$.

Type 2: Similar to type 1 except that the positive maximum occurs at wavelengths shorter than $187 \mathrm{~nm}$.

Type 3: The CD is essentially zero at all wavelengths. 


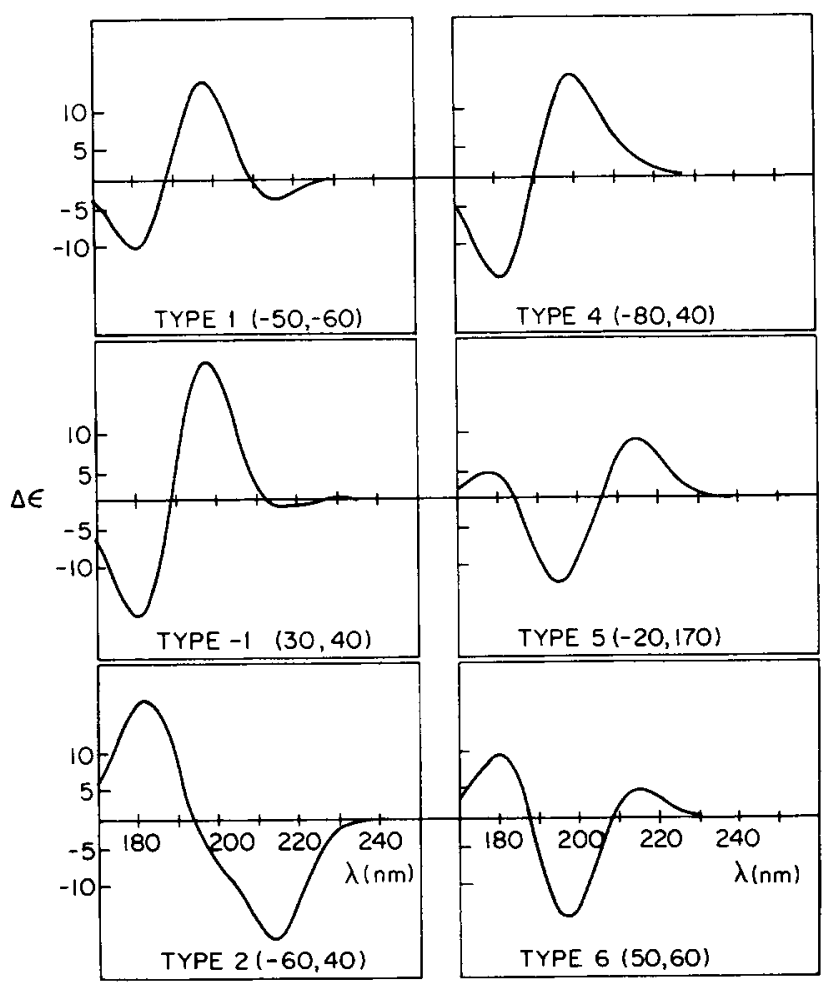

Fig. 2. Examples of types of dipeptide CD spectra classified in the text. The numbers in parentheses are the $(\varphi, \psi)$ values used for the examples.

Type 4: A positive CD at wavelengths greater than $215 \mathrm{~nm}$ followed by a negative $C D$ with a minimum at a wavelength shorter than $185 \mathrm{~nm}$.

Type 5: Similar to type 4 except that the minimum occurs between 185 and $198 \mathrm{~nm}$.

Type 6: Similar to type 4 except that the minimum occurs at wavelengths greater than $198 \mathrm{~nm}$.

A conformational CD map based on this classification is given in Figure 3. Some $\varphi, \psi$ points do not quite fit into the above classification in that small contributions are present in the $230-250 \mathrm{~nm}$ region. These have been designated with a minus sign in Figure 3, indicating that there is a small band in this region which is positive for types 1 and 2 and negative for types 4,5 , and 6 . An example of type - 1 CD spectrum is given in Figure 2.

In order to test the proposal that the CD of a polypeptide chain with an arbitrary sequence of $\varphi, \psi$ angles can be adequately represented by a sum of the corresponding dipeptide CD spectra, calculations were made on oligopeptides containing twenty-eight residues. Then, using the same set of twenty-seven $\varphi, \psi$ angles, the average CD based on dipeptide spectra were determined. Many such calculations were done, but the results are 


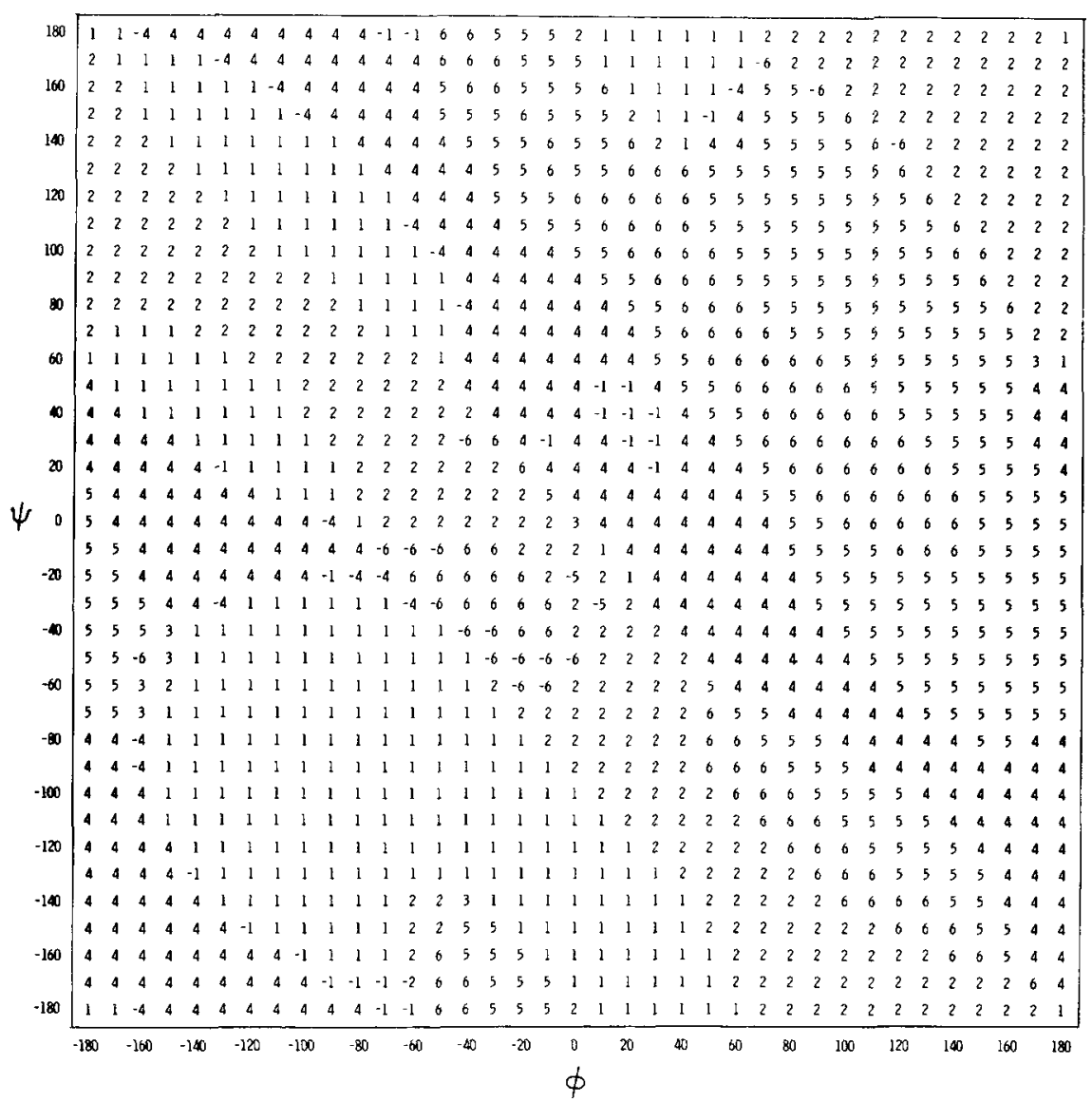

Fig. 3. Dipeptide conformational CD map. Numbers at each $\varphi, \psi$ represented spectral types, and are described in text.

typified by the three examples given in Figures $4 a, b$, and c. In the upper lefthand corner of each figure is a series of points representing the $\varphi, \psi$ angles used for that particular oligopeptide. (One specific sequence of $\varphi, \psi$ is shown, but an examination was made of other sequences and these generally give the same type curve.) The points are restricted to the region of the map represented by $-180 \leqslant \varphi \leqslant 0,0 \leqslant \psi \leqslant 180$, since this is the region most relevant to the unordered chain. In all cases the dipeptide sum spectrum is a reasonable approximation to that of the oligopeptide. The following comparisons are to be noted: (1) the maxima and minima of the oligopeptide in the region of about 170 to $200 \mathrm{~nm}$ are at shorter wavelengths than for the dipeptide spectrum; (2) in both the dipeptide and oligopeptide spectra, the long wavelength negative band (which is a summation of $\pi \pi^{*}$ and $n \pi^{*}$ rotational strengths) is centered near the $n \pi^{*}$ transition (212 $\mathrm{nm}$ in this case); (3) the amplitudes of the oligopeptide bands in the region of 190 to $250 \mathrm{~nm}$ are generally larger than those in the comparable dipeptide spectra. Despite these differences, it appears that the 
dipeptide CD can be satisfactorily used to represent the spectrum of an unordered chain. If we do so, we may expect that the actual chain will have its $\pi \pi^{*}$ peak at a shorter wavelength and that its band may be somewhat more intense.

\section{RESULTS AND DISCUSSION}

Having shown that the $\mathrm{CD}$ of a non-regular chain is reasonably well represented by a sum of dipeptide contributions, we have evaluated the $C D$ of an unordered polypeptide chain from

$$
\bar{R}(\lambda)=\frac{\sum_{i} R_{i}(\lambda) \exp \left(-E_{i} / R T\right)}{\sum_{i} \exp \left(-E_{i} / R T\right)}
$$

In this expression: $i$ represents a $\varphi, \psi$ pair, with the summation being taken at $10^{\circ}$ intervals over the range $-180 \leqslant \varphi \leqslant 0,0 \leqslant \psi \leqslant 180$ (a calculation

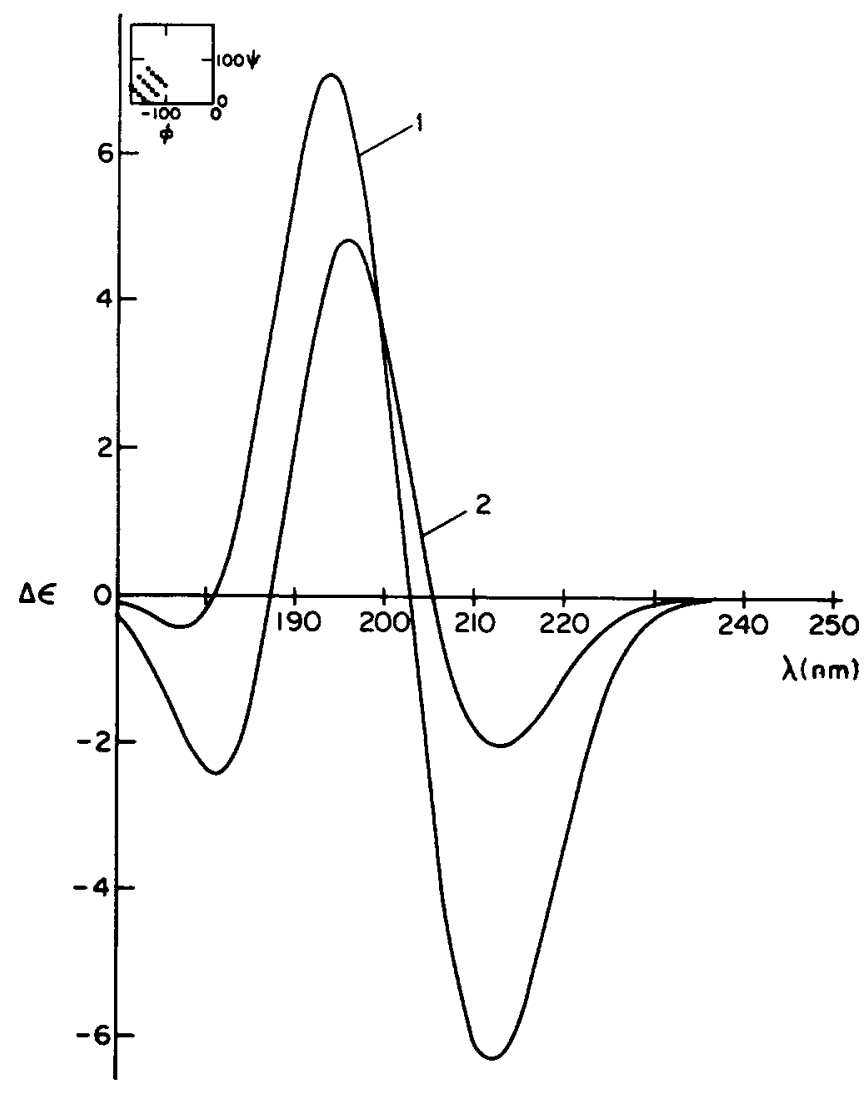

(a)

Fig. 4. (continued) 


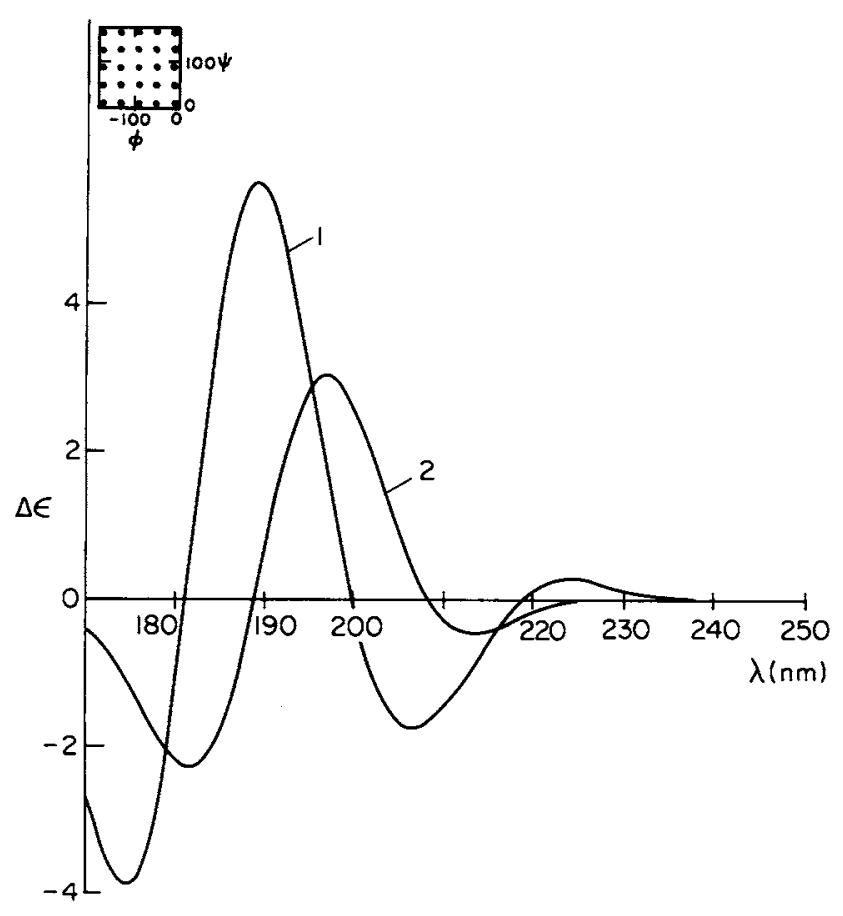

(b)

Fig. 4. (continued)

with $-180 \leqslant \varphi, \psi \leqslant 180$ gave essentially the same result, which is reasonable since most of the low energy conformations are concentrated in the former angular range); $R_{i}(\lambda)$ is the circular dichroism as a function of wavelength for a particular dipeptide, which is shown in Figure 3 and given in detail elsewhere; ${ }^{21} E_{i}$ is the energy of the dipeptide ${ }^{24}$ (the results are similar ${ }^{21}$ if previously published energies ${ }^{25}$ are used); $R=1.986 \times 10^{-3}$ $\mathrm{kcal} / \mathrm{deg}$-mole; and $T=288^{\circ} \mathrm{K}$. The result of such a calculation is shown in Figure 5.

Our calculated CD spectrum of an unordered polypeptide chain, shown in Figure 5, disagrees with previous calculations ${ }^{8,9}$ in not exhibiting any long wavelength positive band. On the other hand, this result is in agreement with detailed experimental studies ${ }^{16,17}$ characterizing this spectrum. The calculated negative band, at about $213 \mathrm{~nm}$, is at a somewhat longer wavelength than that observed..$^{16}$ It must be remembered however, that this is directly related to the location of the $n \pi^{*}$ transition, about which there may be some uncertainty for such structures. In addition, there is evidence ${ }^{17}$ that the negative peak moves to longer wavelengths in the presence of certain salts. These spectra also show that a crossover to positive $\Delta \epsilon$ exists at shorter wavelengths, as predicted in Figure 5 . In view of the fact that the calculated magnitude of the $213-\mathrm{nm}$ band is also 


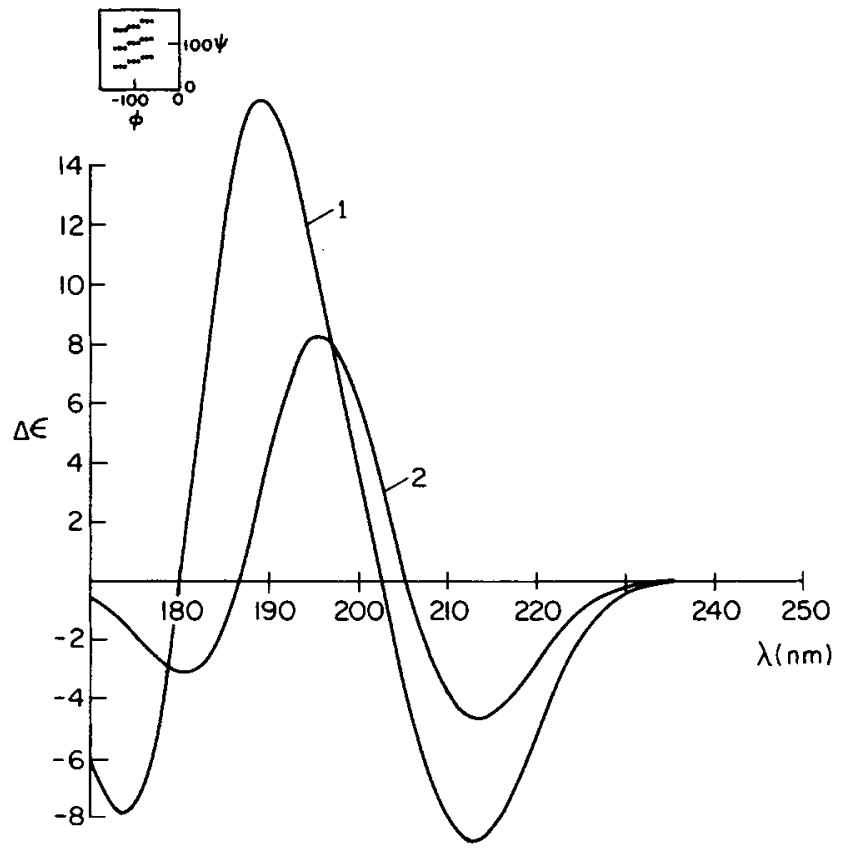

(c)

Fig. 4. Calculated CD spectra of: (1) oligopeptide of twenty-eight residues, (2) the sum of dipeptides with the same twenty-seven pairs of $\varphi, \psi$ angles. The $\varphi, \psi$ angles used are shown in the upper left hand corner of each figure. a), b), and c) represent different sets of $\varphi, \psi$ angles.

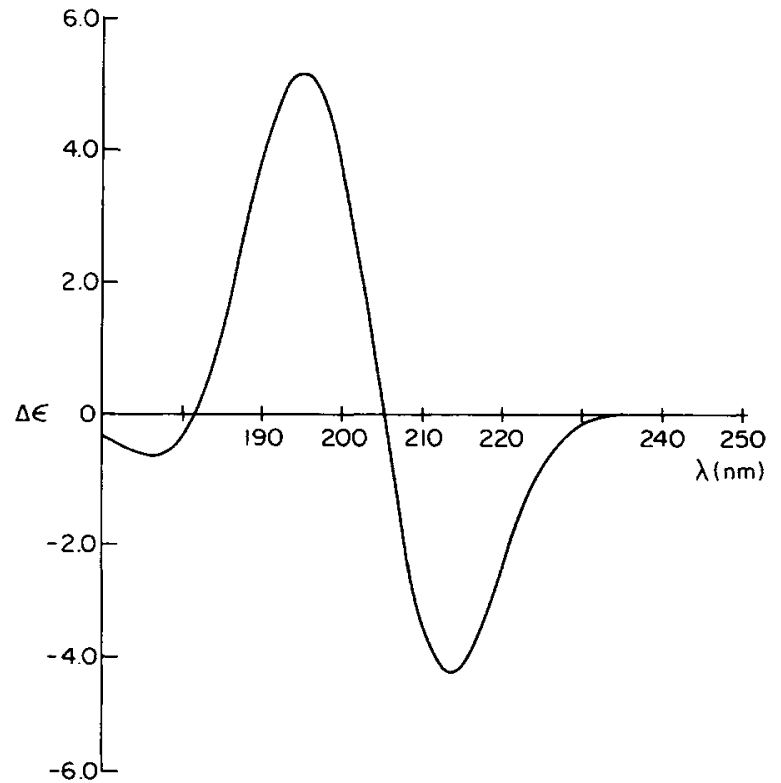

Fig. 5. Calculated CD spectrum of an unordered polypeptide chain. 
in reasonable agreement with that observed for the negative peak, ${ }^{16}$ we feel that the present calculation makes plausible the revised assignments ${ }^{15,16}$ for the CD spectrum of an unordered polypeptide chain.

It has been stressed before, ${ }^{16}$ but it is perhaps worth emphasizing again, that there is probably no single unordered polypeptide chain structure. Different non-regular systems probably sample the $\varphi, \psi$ space in different ways, depending on such factors as interaction with solvent, side chain steric and electrostatic interactions, etc. Our model of the unordered chain is based on a dipeptide energy map in which such factors are minimal. An examination of the CD spectra of a wide variety of unordered polypeptides ${ }^{16,17}$ indicates that the spectrum of Figure 5 is more closely approached the greater is the tendency toward randomization of order in the system. In this sense, the above calculated spectrum could be considered as that of a truly random chain, which is approached more or less closely by real polypeptide systems.

This research was supported by National Science Foundation Grant GB-15682. One of us (E. W. R.) is indebted to the Institute of Science and Technology and to the Macromolecular Research Center for fellowship support.

\section{References}

1. R. W. Woody, J. Chem. Phys., 49, 4797 (1968).

2. E. S. Pysh, J. Chem. Phys., 52, 4723 (1970).

3. E. S. Pysh, Proc. Natl. Acad. Sci., 56, 825 (1966).

4. K. Rosenheck and B. Sommer, J. Chem. Phys., 46, 532 (1967).

5. R. W. Woody, Biopolymers, 8, 669 (1969).

6. P. M. Bayley, E. B. Nielsen, and J. A. Schellman, J. Phys. Chem., 73, 228 (1969).

7. V. Madison and J. Schellman, Biopolymers, 9, 569 (1970).

8. A. E. Tonelli, Macromolecules, 2, 635 (1969).

9. D. Aebersold and E. S. Pysh, J. Chem. Phys., 53, 2156 (1970).

10. V. A. Zubkov, T. M. Birshtein, I. S. Milevskaya, and M. V. Volkenstein, Biopolymers, 10, 2051 (1971).

11. G. Holzwarth and P. Doty, J. Amer. Chem. Soc., 87, 218 (1965).

12. M. Lois Tiffany and S. Krimm, Biopolymers, 6, 1379 (1968).

13. S. Krimm and J. E. Mark, Proc. Natl. Acad. Sci., 60, 1122 (1968).

14. W. B. Rippon and A. G. Walton, Biopolymers, 10, 1207 (1971).

15. M. Lois Tiffany and S. Krimm, Biopolymers, 6, 1767 (1968).

16. M. Lois Tiffany and S. Krimm, Biopolymers, 8, 347 (1969).

17. M. Lois Tiffany, Ph.D. Thesis, University of Michigan, 1970.

18. R. W. Woody and I. Tinoco, J. Chem. Phys., 46, 4927 (1967).

19. E. B. Nielsen and J. A. Schellman, J. Phys. Chem., 71, 2297 (1967).

20. J. A. Schellman and E. B. Nielsen, J. Phys. Chem., 71, 3914 (1967).

21. E. W. Ronish, Ph.D. Thesis, University of Michigan, 1970.

22. H. Basch, M. B. Robin, and N. A. Keubler, J. Chem. Phys., 47, 1201 (1967); ibid., 49, 5007 (1968).

23. F. Quadrifoglio and D. W. Urry, J. Amer. Chem. Soc., 90, 2755 (1967).

24. C. M. Venkatachalam, private communication.

25. D. A. Brant, W. G. Miller, and P. J. Flory, J. Mol. Biol., 23, 47 (1967).

Received February 18, 1972

Revised March 22, 1972 